\title{
CARACTERIZACIÓN SISTEMÁTICA DE LA EFICIENCIA EN LA EDUCACIÓN
}

\section{SYSTEMATIC CHARACTERIZATION OF EFFICIENCY IN EDUCATION}

\author{
Alexander Arévalo Soto, Víctor Giménez García y Diego Prior Jiménez \\ Semillero SEMOSIMA \\ Grupo GISCBA \\ Institución Universitaria Antonio José Camacho \\ Cómo citar este artículo: \\ Arévalo Soto, A., Giménez García, V. y Prior Jiménez, D. (2021). Caracterización sistemática de la eficiencia en la educación. Revista \\ Sapientía, 13(26), 17-31.
}

\section{INTRODUCCIÓN}

En el marco del proyecto de investigación "Eficiencia productiva en la educación. Una aplicación del método StoNED”, que cursa en el Decanto Asociado a Investigaciones de la Institución, el cual tiene como objetivo realizar una evaluación de la eficiencia en el sistema educativo colombiano, se presenta en el siguiente documento, a manera de divulgación, una caracterización sistemática que se realizó para establecer y plantear una revisión de la literatura, sintetizar la evidencia de la investigación (Grant \& Booth, 2009) y mostrar la relevancia y valor agregado del proyecto.

Cabe resaltar que, dicha evaluación de la eficiencia se realiza en pro de complementar los índices utilizados actualmente para la evaluación de las instituciones educativas, los desarrollos académicos y los procesos de enseñanza y aprendizaje que se generan en los estudiantes, dando cumplimiento a los objetivos del Plan Nacional de Desarrollo $(\mathrm{PND})^{1}$ y contribuyendo al diseño de políticas educativas. Lo anterior está planteado bajo la reglamentación del Ministerio de Educación Nacional (MEN), el cual indica que el sistema educativo colombiano lo conforman: la educación inicial, la educación preescolar (atención integral a la primera infancia), la educación básica (primaria cinco grados y secundaria cuatro grados), la educación media (dos grados y culmina con el título de bachiller) y la educación superior (MEN, 2016).

Así mismo, la evaluación busca contemplar el posible sesgo de selección existente, dado por parte de las directivas de las instituciones como consecuencia de la elección de los estudiantes que se presentan a las pruebas SABER 11 que aplica el estado colombiano para evaluar el sistema educativo a través del Instituto Colombiano para el Fomento de la Educación Superior-ICFES² (ICFES, 2016). Por último, trata de visualizar una posible relación de la eficiencia encontrada en el sistema educativo colombiano con la gestión de las secretarías de educación de cada departamento ${ }^{3}$ en este país.

Para abordar la caracterización planteada, se presentarán cuatro enfoques relevantes en la investigación, los cuales tienen que ver con: los estudios relevantes en eficiencia técnica en la educación (a nivel nacional e internacional), los métodos trabajados para medir eficiencia (especialmente los métodos de frontera), el método por utilizar en el estudio y, por último, se documentará una relación entre los partidos políticos y la declaración política de los gobernadores de cada departamento de Colombia (en el periodo administrativo anterior).

\section{DESARROLLO}

A continuación, se presentan cada uno de los desarrollos planteados en este documento:

\footnotetext{
1 El Plan Nacional de Desarrollo es la base de las políticas gubernamentales del presidente de Colombia, sancionado por Ley.

2 Empresa estatal de carácter social y naturaleza especial, vinculada al MEN, que ofrece los servicios de evaluación de la educación en todos sus niveles.
} 


\section{EFICIENCIA TÉCNICA EN LA EDUCACIÓN}

Se presenta una revisión de literatura sistemática, la cual se basó en una búsqueda inicial en la base de datos Web of Science haciendo uso de los campos: título, palabras clave y resumen, introduciendo: "efficiency + educat $^{*}$ or frontier models or govern ${ }^{\star}$. Posteriormente se realizaron dos filtros; uno, por tipo de documento (tomando article) y, dos, por categorias (tomando economics, education educational research, education scientific disciplines, management $y$ operations research management science). Con los resultados obtenidos, se realizó una lectura clasificatoria de los resúmenes y conclusiones de estos para categorizar y definir con cuales se trabajaría. Por último, se identificaron artículos de importancia que, por el año de publicación, no se encontraban en Web of Science; por lo que se hizo una búsqueda secundaria de manera manual en Google Scholar y Scopus.

De esta manera, en la Tabla 1 se realiza una primera síntesis de una serie de estudios relevantes en cuanto a eficiencia técnica en la educación. El diseño de esta síntesis se realiza teniendo en cuenta los autores que llevaron a cabo el estudio, el año que se publicó el artículo de investigación y el lugar donde se desarrolló el estudio.

\begin{tabular}{|c|c|c|}
\hline \multicolumn{3}{|l|}{ Estudios en eficiencia técnica en la educación } \\
\hline Autores & Año & Lugar \\
\hline Hanushek & 1979 & Estados Unidos \\
\hline Bessent y Bessent & 1980 & Estados Unidos \\
\hline Bessent, Bessent, Kennington y Reagan & 1982 & Estados Unidos \\
\hline Hanushek & 1997 & Estados Unidos \\
\hline Portela y Thanassoulis & 2001 & Reino Unido \\
\hline Teddlie y Reynolds & 2001 & Mundial \\
\hline Worthington & 2001 & Mundial \\
\hline Hanushek & 2002 & Estados Unidos \\
\hline Thanassoulis y Portela & 2002 & Reino Unido \\
\hline Hanushek y Luque & 2003 & Estados Unidos \\
\hline Miranda y Araya & 2003 & Chile \\
\hline Fertig y Wright & 2005 & Mundial \\
\hline Giménez, Prior y Thieme & 2007 & Mundial \\
\hline Agasisti y Dal Bianco & 2009 & Italia \\
\hline De Witte, Thanassoulis, Simpson, Battisti y Charlesworth-May & 2010 & Reino Unido \\
\hline Agasisti y Pérez-Esparrells & 2010 & Italia y España \\
\hline Mendoza & 2011 & Colombia \\
\hline Thanassoulis, Kortelainen, Johnes y Johnes & 2011 & Reino Unido \\
\hline Thieme, Prior, Giménez y Tortosa-Ausina & 2011 & España \\
\hline Becerril-Torres, Álvarez-Ayuso y Nava-Rogel & 2012 & México \\
\hline Thieme, Prior y Tortosa-Ausina & 2013 & Chile \\
\hline Johnes & 2014 & Reino Unido \\
\hline Rodriguez-Murillo & 2014 & Colombia \\
\hline Agasisti & 2014 & Unión Europea \\
\hline Morales & 2016 & Estados Unidos \\
\hline Ibáñez-Martín, Morresiy Delbianco & 2017 & Argentina \\
\hline Melo-Becerra, Ramos-Forero y Hernández-Santamaria & 2017 & Colombia \\
\hline Visbal-Cadavid & 2017 & Colombia \\
\hline Giménez, Thieme, Prior y Tortosa-Ausina & 2017 & Mundial \\
\hline Giménez, Thieme, Prior y Tortosa-Ausina & 2019 & Mundial \\
\hline Agasistit, Egorov, Zinchenko y Leshukov & 2020 & Rusia \\
\hline
\end{tabular}

Tabla 1. Estudios relevantes en eficiencia técnica en la educación Fuente: elaboración propia

${ }^{3}$ En este documento se hará referencia a 32 departamentos y a Santa Fe de Bogotá como distrito capital del país. Así, se hablará de 33 departamentos.
Tal como se muestra en la Tabla 1 , los estudios en esta línea de investigación presentan tres connotaciones de importancia: (i) es una línea de estudio relevante en el mundo entero, (ii) en Colombia se ha trabajado tanto para la educación media como para la educación superior, pero no con el énfasis que se busca realizar en el estudio proyectado y, finalmente, (iii) es una temática de actualidad y de auge en desarrollos investigativos. Lo anterior se da principalmente, ya que, como el sistema educativo juega un rol determinante en el desarrollo económico de los países, la medida de su rendimiento y los factores que lo determinan se convierten en un tema de especial relevancia tanto desde una perspectiva académica como de definición de políticas de gobierno.

\section{METODOLOGÍAS UTILIZADAS PARA MEDIR EFICIENCIA}

En los desarrollos metodológicos que se visualizan en los estudios de eficiencia académica, se presentan distintas técnicas utilizadas para lograr el cometido de medir y analizar la eficiencia técnica en términos de la producción. En general, estas metodologías se pueden esquematizar en dos grandes grupos: los métodos de frontera y los métodos de no frontera (Worthington, 2001).

En primera instancia, están los métodos de no frontera, los cuales no requieren la estimación de una frontera de posibilidades de producción, ya que evalúan el desempeño en forma absoluta, es decir, no se requiere la comparación con otras unidades productivas. Entre estos métodos, los más destacados dentro de la literatura (Peretto, 2016) son:

- Indicadores financieros

- Índice de productividad global

- $\quad$ Función de producción promedio.

La Tabla 2 presenta algunos de los trabajos más reconocidos, que han hecho uso de modelos econométricos a partir de los distintos enfoques existentes, identificando los autores de los estudios y los años en que fueron publicadas las investigaciones. 


\begin{tabular}{|l|l|r|}
\hline \multicolumn{2}{|c|}{ Estudios de eficiencia en educación con modelos econométricos } \\
\hline \multicolumn{1}{|c|}{ Enfoque } & \multicolumn{1}{|c|}{ Autores } & \multicolumn{1}{|c|}{ Año } \\
\hline \multirow{4}{*}{$\begin{array}{l}\text { Análisis de los determinantes del } \\
\text { rendimiento académico }\end{array}$} & Hanushek & 1987 \\
\cline { 2 - 3 } & Parky Kerr & 1990 \\
\cline { 2 - 3 } & Carayol y Matt & 2006 \\
\cline { 2 - 3 } & Li, Chen y Duanmu & 2010 \\
\hline \multirow{4}{*}{$\begin{array}{l}\text { Análisis del desempeño de las instituciones } \\
\text { educativas }\end{array}$} & Wößmann & 2003 \\
\cline { 2 - 3 } & Stinebrickner y Stinebrickner & 2003 \\
\cline { 2 - 3 } & Marvasti & 2005 \\
\cline { 2 - 3 } & Mora, García y Molina & 2016 \\
\hline \multirow{3}{*}{$\begin{array}{l}\text { Análisis del desarrollo social y la } \\
\text { integración con el rendimiento académico }\end{array}$} & Schultz & 1997 \\
\cline { 2 - 3 } & Manski & 2010 \\
\cline { 2 - 3 } & Lomi, Steglich y Torló \\
\hline
\end{tabular}

Tabla 2. Eficiencia en educación con modelos econométricos. Fuente: elaboración propia

Sin embargo, es preciso aclarar que este tipo de estimaciones dejan de lado la posibilidad de identificar la estimación de una frontera de eficiencia, la cual permite identificar una función de producción que relaciona los elementos que intervienen en el modelo.

Es segunda instancia, están los métodos de frontera, los cuales son métodos que se han establecido como fiables, puesto que obtienen una función de producción relacionando los elementos que intervienen (productos e insumos) en las unidades productivas consideradas en la evaluación realizada. La función mencionada determina el límite de posibilidades que se pueden obtener de la producción; por lo que, según lo estipulado por estos métodos, la eficiencia de una unidad productiva está dada por la distancia de separación entre la unidad medida y la frontera calculada.

Es preciso aclarar que, se entiende por eficiencia la comparación entre los valores observados y los óptimos (de los inputs y outputs). Dicha comparación se puede realizar:

- Orientada al output; es decir, la comparación entre el output máximo alcanzable y el realmente alcanzado, para un cierto nivel dado de inputs.

- Orientada al input; es decir, la comparación entre los distintos niveles de inputs dados para el output máximo alcanzable.

Además, se debe tener en cuenta que, cuando el ópti- mo está definido por la función de producción, la medida de eficiencia obtenida se denomina eficiencia técnica. En particular, si la comparación se realiza considerando un óptimo definido en términos de un objetivo económico determinado, la medida de eficiencia obtenida se denomina eficiencia económica (Lovell et al., 1994).

De esta manera, en cuanto a los métodos de frontera, se han destacado dos tipos de técnicas básicas (Murillo-Melchor, 1999; Dong et al., 2014), las cuales son:

- Las paramétricas, que son técnicas que especifican la tecnología tomando a priori una forma funcional conocida.

- Las no paramétricas, que son técnicas que exponen las características de la tecnología mediante supuestos planteados de los elementos de producción.

En la Tabla 3 se presenta un esquema donde se relacionan los métodos de frontera más utilizados en la literatura para medir eficiencia. Cabe resaltar que unos métodos son más utilizados que otros; sin embargo, se presentan un bosquejo general de lo trabajado en esta línea de investigación.

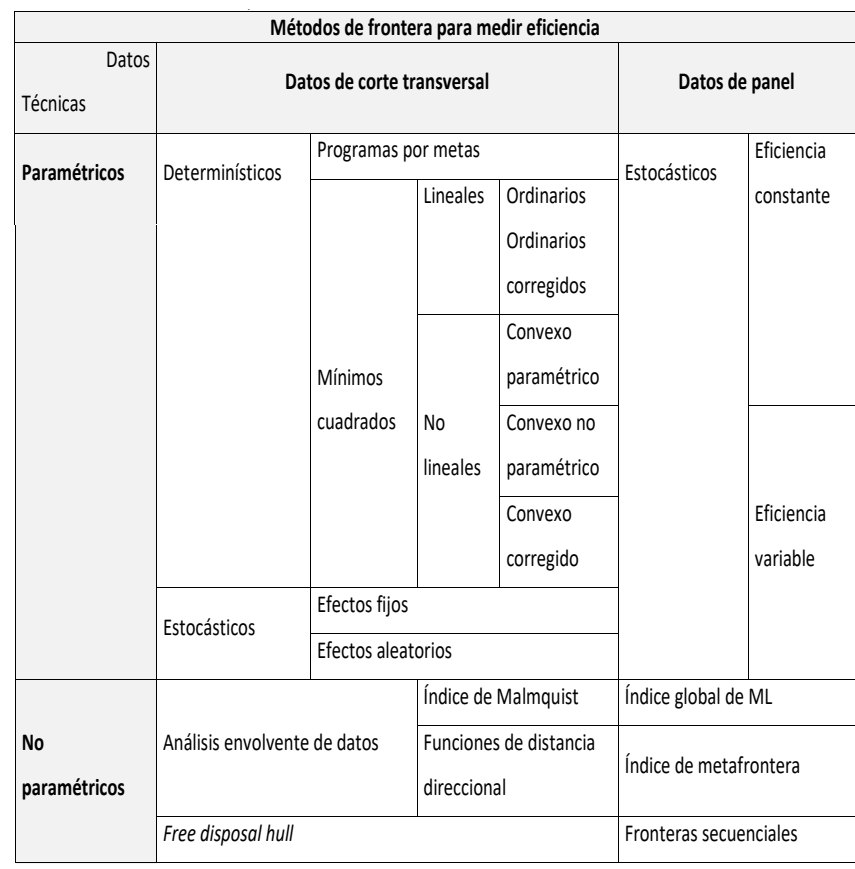

Tabla 3. Métodos de frontera para medir eficiencia Fuente: elaboración propia a partir de Charnes et al. (1997); Murillo-Melchor (1999); Perreto (016). 
De todos los métodos presentados en la Tabla 3, hay dos métodos que sobresalen; uno de cada técnica presentada. En cuanto a la técnica paramétrica, tenemos el modelo de Análisis de Frontera Estocástica (SFA, por sus siglas en inglés Stochastic Frontier Analysis), el cual tiene la ventaja de que permite capturar los errores estocásticos en la realización de la estimación, relacionados principalmente con ineficiencia y factores externos. En cuanto a la técnica no paramétrica, tenemos el Análisis Envolvente de Datos (DEA, por sus siglas en inglés Data Envelopment Analysis), el cual permite estimar la frontera mediante técnicas de programación lineal o no lineal, con la ventaja de que no supone una función determinada para la producción.

La Tabla 4 presenta algunos de los trabajos más reconocidos y recientes, que han hecho uso de modelos de frontera paramétrica y no paramétrica, identificando los autores de los estudios y los años en que fueron publicadas las investigaciones.

\begin{tabular}{|c|c|c|c|}
\hline \multicolumn{4}{|c|}{ Estudios de eficiencia en educación con modelos de frontera } \\
\hline \multicolumn{2}{|c|}{ Modelo de frontera } & Autores & Año \\
\hline \multirow{4}{*}{ Paramétrica } & \multirow{4}{*}{ SFA } & Aigner, Lovell y Schmidt & 1977 \\
\hline & & Manski & 2000 \\
\hline & & Rodríguez-Murillo & 2014 \\
\hline & & Melo-Becerra, Ramos-Forero y Hernández-Santamaría & 2017 \\
\hline \multirow{2}{*}{$\begin{array}{l}\text { No } \\
\text { paramétrica }\end{array}$} & \multirow{2}{*}{ DEA } & Charnes, Cooper y Rhodes & 1978 \\
\hline & & Charnes, Cooper, Lewin y Seiford & 1997 \\
\hline & & Greene & 2008 \\
\hline & & López-Torres y Prior & 2016 \\
\hline & & Giménez, Thieme, Prior y Tortosa-Ausina & 2019 \\
\hline & \multirow{5}{*}{$\begin{array}{l}\text { Índice de } \\
\text { Malmquist }\end{array}$} & Färe, Grosskopf, Norris y Zhang & 1994 \\
\hline & & Lovell & 2003 \\
\hline & & Thanassoulis, Kortelainen, Johnes y Johnes, & 2011 \\
\hline & & Johnson y Ruggiero & 2014 \\
\hline & & Giménez, Thieme, Prior y Tortosa-Ausina & 2017 \\
\hline & \multirow{3}{*}{$\begin{array}{l}\text { Índice de } \\
\text { Metafrontera } \\
\text { Malmquist } \\
\text { Luenberger }\end{array}$} & Oh & 2010 \\
\hline & & Thieme, Prior y Tortosa-Ausina & 2013 \\
\hline & & Cordero, Santín \& Simancas & 2016 \\
\hline
\end{tabular}

Tabla 4. Eficiencia en educación con modelos de frontera Fuente: elaboración propia

\section{METODOLOGÍA POR UTILIZAR PARA MEDIR LA EFICIENCIA}

En el desarrollo del proyecto planteado se ha de trabajar con una metodología que sería innovadora en el sector educativo, denominada Stochastic Non-smooth Envelopment of Data (StoNED), desarrollada por Kuosmanen y Kortelainen (2012). La Tabla 5 presenta diversos estudios en que se ha utilizado el método StoNED, identificando el sector trabajado, los autores de los estudios y los años en que fueron publicadas las investigaciones.

\begin{tabular}{|l|l|r|}
\multicolumn{1}{|c|}{ Sector } & \multicolumn{1}{|c|}{ Autores } & \multicolumn{1}{c|}{ Año } \\
\hline \multirow{4}{*}{ Energía eléctrica } & Kuosmanen, Saastamoinen y Sipiläinen & 2013 \\
\cline { 2 - 3 } & Li, Kopsakangas-Savolainen, Xiao, Tian, Yang y Wang & 2016 \\
\cline { 2 - 3 } & Kuosmanen & 2012 \\
\cline { 2 - 3 } & Cheng, Bjørndal y Bjørnda & 2014 \\
\cline { 2 - 3 } & Dai y Kuosmanen & 2014 \\
\hline \multirow{2}{*}{ Agrícola } & Shen y Lin & 2017 \\
\cline { 2 - 3 } & Chaovanapoonphol y Somyana & 2018 \\
\hline \multirow{2}{*}{ Finanzas } & Eskelinen y Kuosmanen & 2020 \\
\cline { 2 - 3 } & Nguyen, Prior y Van Hemmen & 2015 \\
\hline \multirow{2}{*}{ Academia } & Louca y Demosthenous & \\
\hline \multirow{2}{*}{ Tabla 5. Eficiencia con modelo StoNED. } \\
\cline { 2 - 3 }
\end{tabular}

Esta metodología trata de un modelo de frontera semiparamétrico que combina la frontera no paramétrica tipo DEA, satisfaciendo la monotonicidad y la concavidad, con el término de error compuesto homocedástico estocástico logrado en los modelos paramétricos tipo SFA. Esta metodología se plantea en cuatro pasos (Kuosmanen \& Kortelainen, 2012), aunque no necesariamente se deben ejecutar todos, los cuales se presentan detalladamente a continuación.

\section{CÁLCULO DE LA REGRESIÓN}

Si los resultados observados están sujetos a ineficiencia asimétrica, entonces el supuesto de media $\operatorname{cero}\left(E\left(\varepsilon_{i}\right)\right.$ $=0)$ de análisis de regresión se viola. De hecho, 


$$
E\left(\varepsilon_{i}\right)=E\left(v_{i}-u_{i}\right)=-E\left(u_{i}\right)=-\mu<0,
$$

debido al término asimétrico de ineficiencia no negativa. Por lo tanto, el estimador de los mínimos cuadrados convexos no paramétricos (CNLS, por sus siglas en inglés convex nonparametric least squares) ya no es un estimador consistente de la función de producción frontera $f$.

La regresión de los CNLS estima la media condicional, por lo tanto, se define la función media condicional $g$ como:

$$
g\left(\boldsymbol{x}_{\boldsymbol{i}}\right)=E\left(y_{i} \mid \boldsymbol{x}_{i}\right)=f\left(\boldsymbol{x}_{i}\right)-E\left(u_{i}\right)=f\left(\boldsymbol{x}_{i}\right)-\mu .
$$

Así, si el término de ineficiencia aleatorio $u$ es independiente de las entradas $x_{i}$, entonces el estimador $\hat{g}_{C N L S}\left(\chi_{i}\right)$ es un estimador insesgado y consistente de la función $g$. Si el término de ineficiencia $u$ tiene una varianza constante (es decir, el término de ineficiencia $u$ es homocedástico), entonces el valor esperado del término de ineficiencia $u$ es una constante, denotada como $\mu$, la cual puede estimarse con base en los residuos de los CNLS $\left(\widehat{\varepsilon}_{l_{C N L S}}\right)$.

De otro lado, si la varianza de la ineficiencia difiere entre las observaciones (es decir, $E\left(u_{i}\right)$ ya no es una constante $\mu$ ) sería un caso de ineficiencia heterocedástica (Kuosmanen et al., 2015). De esta manera, para determinar si se debe proceder al siguiente paso, es útil probar los datos para detectar cualquier evidencia de ineficiencia, puesto que el residual de los CNLS $\left(\widehat{\varepsilon}_{{ }_{C N L S}}\right)$ consiste en un término de ruido distribuido normalmente y un término de ineficiencia truncado a la izquierda.

\section{ESTIMACIÓN DE LA INEFICIENCIA ESPERADA}

Teniendo en cuenta los residuos de los CNLS $\left(\widehat{\varepsilon}_{l C N L S}\right)$ es posible estimar el valor esperado del término de ineficiencia $\mu=E\left(u_{i}\right)$. Se debe tener en cuenta que si la varianza de la ineficiencia es constante entre instituciones (el supuesto de homocedasticidad), entonces el valor esperado es tomado incondicional y es constante para todas las instituciones. A continuación, se plantean los enfoques paramétricos comúnmente utilizados basados en el método de momentos (Aigner et al. 1977) y estimación de cuasi verosimilitud (Fan et al. 1996).

\section{MÉTODO DE MOMENTOS}

El método de los momentos requiere algunos supuestos adicionales de distribución paramétrica. Las condiciones de momentos son conocidas, al menos, para las distribuciones de ineficiencia semi normal y exponencial, las cuales son comúnmente utilizadas, pero no para todas las distribuciones consideradas en la literatura. De esta manera, se plantea el caso de ineficiencia semi normal $\left(u_{i} \sim N^{+}\left(0, \sigma_{u}^{2}\right)\right) \quad$ y ruido normal $\left(v_{i} \sim N\left(0, \sigma_{v}^{2}\right)\right)$

Así, se pueden calcular los siguientes momentos centrales (bajo los supuestos paramétricos mencionados) como:

$$
\begin{gathered}
\widehat{M}_{1}=\frac{\sum_{i=1}^{n}\left(\widehat{\varepsilon}_{l C N L S}\right)}{n-1}=0, \\
\widehat{M}_{2}=\frac{\sum_{i=1}^{n}\left(\widehat{\varepsilon}_{l_{C N L S}}\right)^{2}}{n-1} ; M_{2}=\left(\frac{\pi-2}{\pi}\right) \sigma_{u}^{2}+\sigma_{v}^{2}, \\
\widehat{M_{3}}=\frac{\sum_{i=1}^{n}\left(\widehat{\varepsilon}_{l C N L S}\right)^{3}}{n-1} ; M_{3}=\sqrt{\frac{2}{\pi}}\left(1-\frac{4}{\pi}\right) \sigma_{u}^{3},
\end{gathered}
$$

Por lo que las estimaciones para $\sigma_{u}$ y $\sigma_{v}$ son:

$$
\begin{gathered}
\widehat{\sigma_{u}}=\sqrt[3]{\frac{\widehat{M_{3}}}{\sqrt{\frac{2}{\pi}}\left(1-\frac{4}{\pi}\right)}}, \\
\widehat{\sigma_{v}}=\sqrt{\widehat{M_{2}}-\left(\frac{\pi-2}{\pi}\right) \sigma_{u}^{2} .}
\end{gathered}
$$

\section{ESTIMACIÓN DE CUASI VEROSIMILITUD}

Este método consiste en aplicar el método estándar de máxima verosimilitud para estimar los parámetros $\sigma_{u} \mathrm{y}$ $\sigma_{v}$, tomando la forma de la curva CNLS como se da (parametrizando así los coeficientes de la frontera). Se debe tener en cuenta que la función de cuasi verosimilitud se puede establecer como una función de un solo parámetro, la razón $\lambda=\frac{\sigma_{u}}{\sigma_{v}}$ Fan et al. (1996),
de la siguiente manera: 


$$
\ln L(\lambda)=-n \ln \hat{\sigma}+\sum_{i=1}^{n} \ln \Phi\left(-\frac{\widehat{\varepsilon_{l}} \lambda}{\hat{\sigma}}\right)-\frac{1}{2 \hat{\sigma}^{2}} \sum_{i=1}^{n} \widehat{\varepsilon}_{l}^{2},
$$

Donde $\Phi$ denota la función de distribución acumulada de la distribución normal estándar $N(0,1)$. Así, se obtiene:

$$
\begin{gathered}
\widehat{\varepsilon}_{l}=\widehat{\varepsilon}_{\varepsilon_{C N L S}}-\frac{\sqrt{2} \lambda \hat{\sigma}}{\left(\pi\left(1+\lambda^{2}\right)\right)^{\frac{1}{2}}}, \\
\hat{\sigma}=\left(\frac{\frac{1}{n} \sum_{i=1}^{n} \widehat{\varepsilon}_{l C N L S}^{2}}{1-\frac{2 \lambda^{2}}{\pi(1+\lambda)}}\right)^{\frac{1}{2}}
\end{gathered}
$$

Ahora, sustituyendo $\widehat{\varepsilon}_{l}$ y $\hat{\sigma}$ en In $L(\lambda)$ y maximizando la función de cuasi verosimilitud, se logran obtener los respectivos $\hat{\lambda}$ los cuales se sustituyen en $\widehat{\varepsilon}_{l}$ y $\hat{\sigma}$ obteniendo estas estimaciones. Por último, una vez se tengan estas estimaciones, se pueden calcular las estimaciones para $\sigma_{u}$ y $\sigma_{v}$ con las expresiones:

$$
\widehat{\sigma_{u}}=\frac{\hat{\sigma} \hat{\lambda}}{1+\lambda} \quad \mathrm{y} \quad \widehat{\sigma_{v}}=\frac{\hat{\sigma}}{1+\lambda}
$$

De esta manera se pueden calcular los estimadores para estimar $\mu$.

\section{ESTIMANDO LA FUNCIÓN DE PRODUCCIÓN FRONTERA}

Con los datos obtenidos en los pasos 1 y 2 es posible encontrar la producción frontera como la suma de $f\left(x_{i}\right)=g\left(x_{i}\right)+\mu$. Sin embargo, dado que el estimador CNLS solo es único para los vectores de entrada observados, $x_{i}(i=1, \ldots, n)$, se hace uso de lo propuesto por Kuosmanen y Kortelainen (2012), para estimar valores de $\hat{\phi}\left(x_{i}\right)$ como:

$$
\widehat{\phi}_{\min S t O N E D}(\boldsymbol{x})=\min _{\alpha, \boldsymbol{\beta}}\left\{\alpha+\boldsymbol{\beta}^{\prime} \boldsymbol{x} \mid \alpha+\boldsymbol{\beta}^{\prime} \boldsymbol{x}_{\boldsymbol{i}} \geq \hat{f}\left(\boldsymbol{x}_{\boldsymbol{i}}\right), \forall i=1, \ldots, n\right\}
$$

\section{ESTIMACIÓN DE INEFICIENCIAS ESPECÍFICAS}

Bajo el supuesto que la varianza de la ineficiencia es constante entre las unidades (el supuesto de homocedasticidad), entonces el valor esperado es tomado incondicional y es constante para todas las unidades, el cual ha sido complicado con el ruido. Con solo una observación de este proceso, es imposible extraer el nivel de ineficiencia específico de la institución, pero al usar el conjunto de todos los residuos estimados podemos comparar el residuo específico de una institución para dar una idea del rendimiento de la institución.

Además, bajo el supuesto de un término de error distribuido normalmente y un término de ineficiencia distribuido aproximadamente normal, Kuosmanen y Kortelainen (2012) derivan una fórmula para la distribución condicional de ineficiencia $u_{i}$, dado $\varepsilon_{i}$ (basada en Jondrow et al., 1982), y proponen el estimador de ineficiencia como la media condicional $E\left(u_{i} \mid \varepsilon_{i}\right)$. Por lo tanto, dados los parámetros estimados $\widehat{\sigma_{u}}$ y $\widehat{\sigma_{v}}$, se calcula el valor condicional esperado de ineficiencia como:

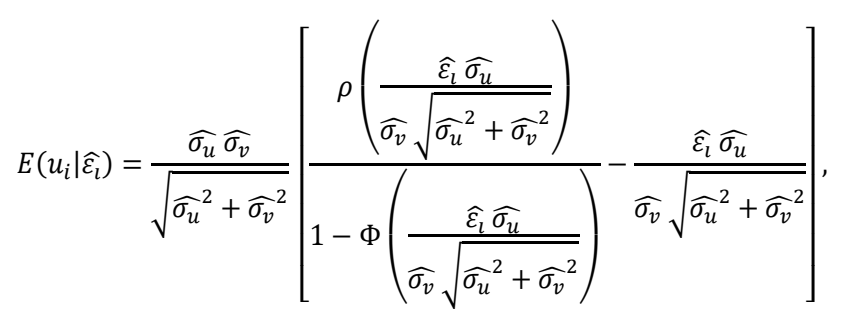

Donde $\rho$ es la función de densidad de la distribución normal estándar $N(0,1), \Phi$ es la función de distribución acumulada correspondiente, y $\widehat{\varepsilon}_{l}=\widehat{\varepsilon}_{l C N L S}-\widehat{\sigma_{u}} \sqrt{\frac{2}{\pi}}$ es el estimador del término de error compuesto. Nótese que la correlación de rango de los residuales CNLS $\left(\widehat{\varepsilon}_{l_{C N L S}}\right)$ y las estimaciones de ineficiencia es igual a uno; que, para los efectos de las clasificaciones de eficiencia relativa, son suficientes. 


\section{VARIABLES PARA TENER EN CUENTA}

De acuerdo con la revisión de literatura realizada y la información recopilada, en cuanto a la eficiencia en la educación, es un común denominador hacer uso de los puntajes de las pruebas tomadas como objeto de estudio como output (Lopez-Torres \& Prior, 2016; Giménez et al., 2017; Sagarra, Mar-Molinero \& Agasisti, 2017; Giménez et al., 2019); por tanto, siguiendo esta línea de estudio y basados en el argumento de integrar más de una asignatura para lograr estudios más confiables, se propone tomar como output los resultados globales de los estudiantes en las pruebas, por institución educativa.

En cuanto a los inputs que se proponen para este estudio, acordes con las variables utilizadas como inputs en la línea de eficiencia en la educación que se pueden encontrar en la literatura, son los siguientes: (i) Cantidad de alumnos matriculados en la institución educativa (Thieme et al., 2013; Crespo-Cebada et al., 2014; De Witte \& López-Torres, 2015), que enmarca características propias de la institución con la calidad educativa de la misma. (ii) Cantidad de docentes (Cordero et al., 2016; Lopez-Torres \& Prior, 2016; Haelermans \& Ruggiero, 2017; Tran \& Villano, 2018), teniendo en cuenta los que son o no de planta, así como directivos de la institución. (iii) Equipos electrónicos (Perelman \& Santín, 2011; Portela et al., 2013; Agasisti, 2014), incluyendo todos los dispositivos electrónicos con que cuenta la institución. Finalmente, un (iv) índice económico, social y cultural (Thieme et al., 2013; Crespo-Cebada et al., 2014; Cordero et al., 2017; Giménez et al., 2017), como factores de entorno y variables ambientales de los estudiantes e instituciones educativas relacionadas con las zonas, afinidades políticas de las poblaciones y la seguridad o situaciones de conflicto en los departamentos (Aparicio et al., 2017).

\section{ESTRUCTURA PARA AFINIDAD POLÍTICA}

Por último, pero no menos importante, está la consideración de la afinidad política o ideológica de los dirigentes (gobernadores) de los departamentos. En este sentido se plantea una relación entre eficiencia educativa y gobierno, en la cual se identifica los distintos puntos de vista que han dado los investigadores en dicho contexto; mostrando cómo la medición de la eficiencia en la educación puede connotar distintos efectos en el ámbito gubernamental, haciendo uso de dicha medida como un instrumento aplicable o argumentativo en las acciones y decisiones gubernamentales.

En este orden de ideas, a continuación, se establecen cuatro enfoques discutidos en cuanto a esta relación: (i) Instrumentos de desarrollo social (Koçak, Türe \& Atan, 2019; Mazurek \& Mielcová, 2019; Johnes \& Virmani, 2019; Nix et al., 2020; Rymarzak \& Marmot, 2020; Agasisti et al, 2020; Padilla, Cachanosky \& Beck, 2020), enfocado a productividad de las instituciones, desarrollo académico, educación y resultados versus factores socioeconómicos, reformas educativas y desarrollo regional. (ii) Ajuste financiero (Haug \& Blackburn, 2017; Wang, 2019; Andrejević-Panić \& Lozanov-Crvenković, 2019), enfocado a instituciones, formación y rendimiento educativo versus financiación y repartición de recursos. (iii) Legislación política (OECD/PISA, 2016; Salinas \& Solé-Ollé, 2018; Sulis, Giambona \& Porcu, 2020; Du Plessis, 2020), enfocada a competencias y pruebas de los estudiantes versus procesos, resultados e implicaciones políticas y legislativas. (iv) Competitividad, categorización y profesorado (Kumar \& Thakur, 2019; Silander \& Stigmar, 2019; Brzezicki, Pietrzak \& Cieciora, 2020; Moncayo-Martínez et al, 2020; Salas-Velasco, 2020), enfocado a planta docente, recursos educativos y desempeño institucional versus competitividad, formación docente y restricciones gubernamentales.

Según lo muestra la Tabla 8, se especifica tanto el partido político, como la declaración política de cada 


\section{4 | Sapıentia Vol. 13, №. 26, noviembre 202I. pág. 17-3I ISSN 1909-08II}

uno de los gobernadores de los departamentos de Colombia en el periodo 2016 a 2019 (periodo anterior de gobernadores departamentales).

\begin{tabular}{|l|l|l|}
\hline Departamento & Partido político & Declaración política \\
\hline Amazonas & Cambio Radical & Gobierno \\
\hline Antioquia & Liberal - Cambio Radical & Gobierno \\
\hline Arauca & Cambio Radical & Gobierno \\
\hline Atlántico & Liberal & Independiente \\
\hline Bogotá & Cambio Radical & Gobierno \\
\hline Bolivar & Liberal & Independiente \\
\hline Boyacá & Alianza Verde - Liberal & Oposición - Independiente \\
\hline Caldas & La U - Cambio Radical & Gobierno - Gobierno \\
\hline Caquetá & Liberal & Independiente \\
\hline Casanare & Centro Democrático & Oposición \\
\hline Cauca & Liberal - Cambio Radical - AICO & Independiente - Gobierno - Independiente \\
\hline Cesar & La U & Gobierno \\
\hline Choco & Liberal - La U - AICO & Independiente - Gobierno - Independiente \\
\hline Córdoba & La U - Liberal - AICO & Gobierno - Independiente - Independiente \\
\hline Cundinamarca & Cambio Radical - La U - ASI & Gobierno - Gobierno - Oposición \\
\hline Guainía & Liberal - Cambio Radical & Independiente - Gobierno \\
\hline Guaviare & AlCO & Independiente \\
\hline Huila & Cambio Radial - ASI & Gobierno - Oposición \\
\hline La Guajira & Cambio Radical & Gobierno \\
\hline Magdalena & Cambio Radical & Gobierno \\
\hline Meta & Liberal - Alianza Verde & Independiente - Oposición \\
\hline Nariño & Somos Nariño & Independiente \\
\hline Norte de Sant. & La U - Liberal & Gobierno - Independiente \\
\hline Putumayo & Alianza Verde - La U - Cambio Radical & Oposición - Gobierno - Gobierno \\
\hline Quindío & Liberal - Conservador & Independiente - Oposición \\
\hline Risaralda & Conservador & Oposición \\
\hline San Andrés & Liberal - La U & Independiente - Gobierno \\
\hline Santander & Liberal & Independiente \\
\hline Sucre & Cambio Radical & Gobierno \\
\hline Tolima & Conservador - Centro Democrático & Oposición - Oposición \\
\hline Valle & La U & Gobierno \\
\hline Vaupés & Cambio Radical & \\
\hline Vichada & La U - Cambio Radical & Gobierno \\
\hline
\end{tabular}

Tabla 6. Partidos y declaración política de gobernadores. Fuente: elaboración propia (a partir del CNE de Colombia)

A priori, se debe identificar que puede no haber mayor claridad en la correspondencia de las ideologías políticas con respecto al impacto en la eficiencia académica de las instituciones educativas objeto de estudio; ya que, históricamente, tanto los departamentos líderes en eficiencia como los dos departamentos con menor rendimiento son partidos afines al Gobierno Nacional en la primera mitad del periodo en mención. Sin embargo, se debe tener presente que para la segunda mitad del periodo de estudio el Gobierno Nacional cambió en su partido y declaración política, lo cual hace que las diferencias se puedan demarcar de manera visible, con respecto a la primera mitad del periodo.

El planteamiento anterior cobra sentido al hacer un análisis de las elecciones regionales y las coaliciones resultantes en ese momento, donde se identifican las mecánicas multipartidistas de partidos fragmentados a lo largo de la historia. Este comportamiento es más notorio en los comicios para la gobernación, donde son mucho más significativos desde el punto de vista de la asignación y la distribución de recursos (patronazgo, clientelismo, etc.) (Milanese et al., 2016). Más aún, en vista de los próximos comicios presidenciales y legislativos de 2018, teniendo en cuenta que los periodos administrativos locales $y$ nacionales no coinciden.

Para finalizar, se debe plantear otro punto de vista en el que es importante remarcar que la referencia de estas mecánicas puede ser circunstancial y temporal. En este escenario se ajusta a la definición del partido como franquicia en la que confluyen coyunturalmente líderes provenientes de distintos orígenes (inclusive ideologías) como consecuencia de la necesidad de un aval como criterio formal necesario para lanzarse a los comicios de una determinada región o país (Milanese \& Jaramillo, 2015). Esta idea genera una discusión mayor en cuanto a la linealidad de las afinidades políticas de los dirigentes en relación con los resultados que se puedan obtener en mediciones, como la de eficiencia, en el caso de instituciones educativas.

\section{CONCLUSIONES}

Se logró presentar de manera clara y amplia distintos enfoques correspondientes a la caracterización sistemática de la literatura presente y la línea que se busca establecer en la investigación en curso. A continuación, se enumeran distintas conclusiones correspondientes con lo desarrollado en este documento: 
- De manera detallada, se presentan los estudios relacionados en eficiencia técnica desde sus inicios, con las investigaciones que marcaron un punto de inicio en esta línea de estudio, hasta la actualidad, con los trabajos más recientes; mostrando la relevancia e importancia de la temática.

- $\quad$ Se expone un amplio bosquejo referente a las metodologías que se han utilizado, y que actualmente se utilizan, en la medición de eficiencia técnica; particularmente, en el desarrollo de trabajos del ámbito educativo.

- $\quad$ Se explica, clara y detalladamente, el proceso que sigue la metodología que se planea desarrollar en el trabajo de investigación. Se aclara que es una metodología que actualmente, al conocimiento de los autores, no tiene un desarrollo enfocado a la educación; por lo que sería un trabajo pionero en este aspecto, lo que abriría una nueva línea de desarrollo investigativo en educación y complementaría los argumentos para utilizarse en otras líneas de investigación.

- $\quad$ Se pone en escena el ambiente político de los esquemas departamentales, en el principio del periodo tomado para el desarrollo del estudio; enmarcando los partidos políticos, así como las declaraciones políticas de cada gobernación de Colombia. Este ambiente presentado pone al descubierto distintos escenarios que pueden llegar a jugar un papel importante en el planteamiento de las ideologías políticas de cada secretaría de educación de las gobernaciones, en relación con los resultados que se pueden obtener de la medición de la eficiencia.

De esta manera, y finalmente, con base en lo presentado en este documento se esboza suficiente evidencia académica y de investigación para el desarrollo del estudio mencionado como objetivo del proyecto referenciado. Todo lo anterior, establecido en el objetivo de este documento de divulgación.

\section{REFERENCIAS BIBLIOGRÁFICAS}

Agasisti, T. \& Dal Bianco, A. (2009). Reforming the university sector: Effects on teaching efficiency evidence from Italy. Higher Education, 57(4), 477-498.

Agasisti, T. \& Pérez-Esparrells, C. (2010). Comparing efficiency in a cross-country perspective: The case of Italian and Spanish state universities. Higher Education, 59(1), 85-103.

Agasisti, T. (2014). The efficiency of public spending on education: An empirical comparison of EU countries. European Journal of Education, 49(4), 543-557.

Agasisti, T., Egorov, A., Zinchenko, D. \& Leshukov, O. (2020). Efficiency of regional higher education systems and regional economic short-run growth: Empirical evidence from Russia. Industry and Innovation, 28(4), 507-534.

Aigner, D.; Lovell, C. \& Schmidt, P. (1977). Formulation and estimation of stochastic frontier production function models. Journal of Econometrics, 6(1), 21-37.

Andrejević-Panić, A. \& Lozanov-Crvenković, Z. (2019) Analysis of higher education indicators coherency in central and eastern Europe. Business Systems Research Journal, 10(2), 6-17.

Aparicio, J., Crespo-Cebada, E., Pedraja-Chaparro, \& Santín, D. (2017). Comparing school ownership performance using a pseudo-panel databasE: A Malmquist-type index approach. European Journal of Operational Research, 256(2), 533-542. 
Becerril-Torres, O. U., Álvarez-Ayuso, I. C. \& Nava -Rogel, R. M. (2012). Frontera tecnológica y eficiencia técnica de la educación superior en México. Revista Mexicana de Investigación Educativa, 17(54), 793-816.

Bessent, A. \& Bessent, W. (1980). Determining the comparative efficiency of schools through data envelop-ment analysis. Educational Administra tion Quar - terly, 16 (2), 57-75.

Bessent, A., Bessent, W., Kennington, J. \& Reagan, B. (1982). An application of mathematical programming to assess productivity in the Houston independent school district. Management Science, 28(12), 1355-1367.

Brzezicki, Ł., Pietrzak, P. \& Cieciora, M. (2020). The total efficiency of teaching activity of polish higher edu cation institutions. Foundations of Management, 12(1), 19-30.

Carayol, N. \& Matt, M. (2006). Individual and collective determinants of academic scientists' productivity. Information Economics and Policy, 18(1), 55-72.

Chaovanapoonphol, Y. \& Somyana, W. (2018) Production efficiency of maize farmers under contract farming in Laos PDR. Kasetsart Journal of Social Sciences, 41(1), 104-109.

Charnes, A., Cooper, W. \& Rhodes, E. (1978). Measuring the efficiency of decision making units. European Journal of Operational Research, 2(6), 429-444.

Charnes, A., Cooper, W., Lewin, A. Y. \& Seiford, L. M. (1997). Data envelopment analysis theory, methodology and applications. Journal of the Operational Research Society, 48(3), 332-333

Cheng, X., Bjørndal, E. \& Bjørndal, M. (2014). Cost efficiency analysis based on the DEA and StoNED models: case of Norwegian electricity distribution companies. In 11th International Conference on the European Energy Market (EEM14), 1-6.

Cordero, J. M., Prior, D. \& Simancas, R. (2016). A comparison of public and private schools in Spain using robust nonparametric frontier methods. Central European Journal of Operations Research, 24(3), 659-680.

Crespo-Cebada, E., Pedraja-Chaparro, F. \& Santín, D. (2014). Does school ownership matter? An unbiased efficiency comparison for regions of Spain. Journal of Productivity Analysis, 41(1), 153-172.

Dai, X. \& Kuosmanen, T. (2014). Best-practice bench-. marking using clustering methods: Application to energy regulation. Omega, 42(1), 179-188.

De Witte, K., Thanassoulis, E., Simpson, G., Battisti, G. \& Charlesworth-May, A. (2010). Assessing pupil and school performance by non-parametric and parametric techniques. Journal of the Operational Research Society, 61(8), 1224-1237.

De Witte, K. \& López-Torres, L. (2015). Efficiency in education: A review of literature and a way forward. Journal of the Operational Research Society, 68(4), 339-363.

Dong, Y., Hamilton, R. \& Tippett, M. (2014). Cost efficiency of the chinese banking sector: A comparison of stochastic frontier analysis and data envelopment analysis. Economic Modelling, 36, 298-308.

Du Plessis, A. (2020). The emergence of decentralised centralism in the South African education governance system. Journal of Southern African Studies, 46(1), 165-183.

Eskelinen, J. \& Kuosmanen, T. (2013). Intertemporal efficiency analysis of sales teams of a bank: Stochastic semi-nonparametric approach. Journal 
of Banking \& Finance, 37(12), 5163-5175.

Färe, R., Grosskopf, S., Norris, M. \& Zhang, Z. (1994). Productivity growth, technical progress, and efficiency change in industrialized countries. The American Economic Review, 84(1), 66-83.

Fertig, M. \& Wright, R. E. (2005). School quality, educational attainment and aggregation bias. Economics Letters, 88(1), 109-114.

Giménez, V., Prior, D. \& Thieme, C. (2007). Technical efficiency, managerial efficiency and objetive-setting in the educational system: An international comparison. The Journal of the Operational Research Society, 58(8), 996-1007.

Giménez, V., Thieme, C., Prior, D. \& Tortosa-Ausina, E. (2017). An international comparison of educational systems: A temporal analysis in presence of bad outputs. Journal of Productivity Analysis, 47(1), 83-101.

Giménez, V., Thieme, C., Prior, D. \& Tortosa-Ausina, E. (2019). Comparing the performance of national educational systems: Inequality versus achievement? Social Indicators Research, 141(2), 581-609.

Grant, M. \& Booth, A. (2009). A typology of reviews: An analysis of 14 review types and associated methodologies. Health Information \& Libraries Journal, 26(2), 91-108.

Haelermans, C. \& Ruggiero, J. (2017). Non-parametric estimation of the cost of adequacy in education: the case of Dutch schools. Journal of the Operational Research Society, 68(4), 390-398.

Hanushek, E. A. (1979). Conceptual and empirical issues in the estimation of educational production functions. Journal of Human Resources, 14(3), 351-388.
Hanushek, E. A. (1987). Educational production functions. Economics of Education, 2(1), 33-42.

Hanushek, E. A. (1997). Assessing th effects of school resources on student performance: An update. Educational Evaluation and Policy Analysis, 19(2), 141-164.

Hanushek, E. A. (2002). Publicly provided education. Handbook of Public Economics, 4(2), 2045-2141.

Hanushek, E. A., \& Luque, J. A. (2003). Efficiency and equity in schools around the world. Economics of Education Review, 22(5), 481-502.

Haug, A. A. \& Blackburn, V. C. (2017). Government secondary school finances in New South Wales: Accounting for students' prior achievements in a two-stage DEA at the school level. Journal of Productivity Analysis, 48(1), 69-83.

Ibáñez-Martín, M. M., Morresi, S. S. \& Delbianco, F. (2017). Una medición de la eficiencia interna en una universidad argentina usando el método de fronteras estocásticas. Revista de la Educación Superior, 46(183), 47-62.

Instituto Colombiano para la Evaluación de la educación (2016). SABER en breve. 5 ed. ICFES: Santafe de Bogotá, Colombia.

Johnes, J. (2014). Efficiency and mergers in english higher education 1996/7 to 2008/9: Parametric and non-parametric estimation of the multi-input multi-output distance function. The Manchester School, 82(4), 465-487.

Johnes, G. \& Virmani, S. (2019). The efficiency of private and public schools in urban and rural areas: Moving beyond the development goals. International Transactions in Operational Research, 27(4), 1869-1885.

Johnson, A. L. \& Ruggiero, J. (2014). Nonparametric measurement of productivity and efficiency in education. Annals of Operations Research, 221(1), 197-210. 
Koçak, D., Türe, H. \& Atan, M. (2019). efficiency measurement with network DEA: An application to sustainable development goals 4 . International Journal of Assessment Tools in Education, 6(3), 415-435.

Kumar, A. \& Thakur, R. R. (2019). Objectivity in performance ranking of higher education institutions using dynamic data envelopment analysis. International Journal of Productivity and Performance Management, 68(4), 774-796..

Kuosmanen, T. \& Kortelainen, M. (2012). Stochastic non-smooth envelopment of data: Semi-parametric frontier estimation subject to shape constraints. Journal of Productivity Analysis, 38(1), 11-28.

Kuosmanen, T. (2012). Stochastic semi-nonparametric frontier estimation of electricity distribution networks: Application of the StoNED method in the Finnish regulatory model. Energy Economics, 34(6), 2189-2199.

Kuosmanen, T., Saastamoinen, A. \& Sipiläinen, T. (2013). What is the best practice for benchmark regulation of electricity distribution? Comparison of DEA, SFA and StoNED methods. Energy Policy, 61(1), 740-750.

Li, G., Chen, W. \& Duanmu, J. L. (2010). Determinants of international students' academic performance: A comparison between Chinese and other international students. Journal of Studies in international Education, 14(4), 389-405.

Li, H. Z., Kopsakangas-Savolainen, M., Xiao, X. Z., Tian, Z. Z., Yang, X. Y. \& Wang, J. L. (2016). Cost efficiency of electric grid utilities in China: A comparison of estimates from SFA-MLE, SFA -Bayes and StoNED-CNLS. Energy Economics, 55(1), 272-283.

Lomi, A., Snijders, T. A., Steglich, C. E. \& Torló, V. J. (2011). Why are some more peer than others?
Evidence from a longitudinal study of social networks and individual academic performance. Social Science Research, 40(6), 1506-1520.

López-Torres, L. \& Prior, D. (2016). Centralized allocation of human resources. An application to public schools. Computers \& Operations Research, 73, 104-114.

Louca, C. N. \& Demosthenous, A. (2015). Education and sustainable economic growth: A theoretical approach of measuring efficiency in education. International Journal of Sustainable Agricultural Management and Informatics, 1(3), 235-246.

Lovell, C. K. (2003). The decomposition of Malmquist productivity indexes. Journal of Productivity Analysis, 20(1), 437-458.

Lovell, C. K., Grosskopf, S., Ley, E., Pastor, J. T., Prior, D. \& Eeckaut, P. V. (1994). Linear programming approaches to the measurement and analysis of productive efficiency. Top, 2(2), 175-248.

Manski, C. F. (2000). Economic analysis of social interactions. Journal of Economic Perspectives, 14(3), 115-136.

Marvasti, A. (2005). US academic institutions and perceived effectiveness of foreign-born faculty. Journal of Economic Issues, 39(1), 151-176.

Mazurek, J. \& Mielcová E. (2019). On the relationship between selected-socio-economic indicators and student performances in the PISA 2015 study. Economy and Management, 22(2), 22-39.

Melo-Becerra, L. A., Ramos-Forero, J. E. \& Hernández -Santamaría, P. O. (2017). La educación superior en Colombia: situación actual y análisis de eficiencia. Revista Desarrollo y Sociedad, 78, 59-111. 
Mendoza, A. M. (2011). Propuesta para la medición del rendimiento académico de los estudiantes de la universidad libre utilizando análisis envolvente de datos (DEA). Revista Academia Libre, 8(9), 77-82.

Milanese, J. P. \& Jaramillo L. E. (2015). Impacto de los factores institucionales del sistema electoral en la fragmentación partidaria. Un análisis de las elecciones para concejos municipales en el Valle del Cauca (1997-2011). Colombia Internacional, 84(2), 43-70.

Milanese, J. P., Abadía, A. A. \& Manfredi, L. (2016). Elecciones regionales en Colombia 2015: Análisis de las nuevas gobernaciones y asambleas departamentales. Revista Uruguaya de Ciencia Política, 25(1), 1-26.

Ministerio de Educación Nacional (2016). Revisión de políticas nacionales de educación: La educación en Colombia (en línea). Recuperado de https:// www.mineducacion.gov.co/1759/articles -356787_recurso_1.pdf

Miranda J. C. \& Araya, L. (2003). Eficiencia económica en las escuelas del MECE/Rural desde la perspectiva del análisis envolvente de datos (DEA). Estudios Pedagógicos, 29, 27-39.

Moncayo-Martínez, L. A., Ramírez-Nafarrate, A. \& Hernández-Balderrama, M. G. (2020). Evaluation of public HEI on teaching, research, and knowledge dissemination by Data Envelopment Analysis. Socio-Economic Planning Sciences, 69(1), 100-718.

Mora, C. D., García, J. A. \& Molina, A. (2016). ¿Dónde está la clave del éxito académico? Un análisis de la relación entre el uso del tiempo y el rendimiento académico. Cultura y Educación: Culture and Education, 28(1), 173-195.

Morales, J. C. (2016). El informe Coleman, 50 años después. Revista de Sociología de la Educación -RASE, 9(1), 9-21.
Murillo-Melchor, C. (1999). An analysis of technical efficiency and productivity changes in Spanish airports using the Malmquist index. International Journal of Transport Economics, 26(2), 271-292.

Nguyen, T. T., Prior, D. \& Van Hemmen, S. (2020). Stochastic semi-nonparametric frontier approach for tax administration efficiency measure: Evidence from a cross-country study. Economic Analysis and Policy, 66(1), 137-153.

Nix, A. N., Jones, T. B., Brower, R. L. \& Hu, S. (2020). Equality, efficiency, and developmental education reform: The impact of SB 1720 on the mission of the Florida college system. Community College Review, 48(1), 55-76.

OECD/UNESCO (2016). Education in Thailand: An OE CD-UNESCO perspective. Paris, France. Reviews of national policies for education.

Oh, D. (2010). A global Malmquist-Luenberger producti vity index. Journal of Productivity Analysis, 34(164), 183-197.

Padilla, A., Cachanosky, N. \& Beck, J. (2020). Immigration and economic freedom: Does education matter? The Journal of Private Enterprise, 35(1), 29-57.

Park, K. H. \& Kerr, P. M. (1990). Determinants of academic performance: A multinomial logit approach. The Journal of Economic Education, 21(2), 101-111.

Peretto, C. (2016). Métodos para medir y evaluar la eficiencia de unidades productivas. Revista de la Escuela de Perfeccionamiento en Investigación Operativa, 24(39), 5-25.

Portela, M. C., Camanho, A. S. \& Keshvari, A. (2013). Assessing the evolution of school performance and value-added: Trends over four years. Journal of Productivity Analysis, 39(1), 1-14. 
Portela, M. C. A. \& Thanassoulis, E. (2001). Decomposing school and school-type efficiency. European Journal of Operational Research, 132(2), 357-373.

Rodriguez-Murillo, I. (2014). Eficiencia de la edcucación superior en Colombia: Un análisis mediante fronteras. Revista CIFE: Lecturas de Economía Social, 16(24), 163-194.

Rymarzak, M. \& Marmot, A. (2020). Higher education estate data accountability: The contrasting experience of UK and Poland. Higher Education Policy, 33(1), 179-194.

Sagarra, M., Mar-Molinero, C. \& Agasisti, T. (2017). Exploring the efficiency of mexican universities: Integrating data envelopment analysis and multidimensional scaling. Omega, 67(1), 123-133.

Salas-Velasco, M. (2020). Does greater school resource allocation improve efficiency in education produc- tion? Performance assessment of Spanish public sector-funded schools. International Journal of Educational Management, 34(5),903-915.

Salinas, P. \& Solé-Ollé, A. (2018). Partial fiscal decentralization reforms and educational outcomes: A difference-in-differences analysis for Spain. Journal of Urban Economics, 107, 31-46.

Schultz, T. P. (1997). Assessing the productive benefits of nutrition and health: An integrated human capital approach. Journal of Econometrics, 77(1), 141-158.

Shen, X. \& Lin, B. (2017). The shadow prices and demand elasticities of agricultural water in China: A StoNED-based analysis. Resources, Conservation and Recycling, 127(1),21-28.

Silander, C. \& Stigmar, M. (2019). Individual growth or institutional development? Ideological perspecti ves on motives behind swedish higher education teacher training. Higher Education, 77(2),265-281.
Stinebrickner, R. \& Stinebrickner, T. R. (2003). Working during school and academic performance. Journal of Labor Economics, 21(2), 473-491.

Sulis, I., Giambona, F. \& Porcu, M. (2020). Adjusted indicators of quality and equity for monitoring the education systems over time. Insights on EU15 countries from PISA surveys. Socio-Economic Planning Sciences, 69(1), 100-718.

Teddlie, C. \& Reynolds, D. (2001). Countering the critics: responses to recent criticisms of school effectiveness research. School Effectiveness and School Improvement, 12(1), 41-82.

Thanassoulis, E. \& Portela, M. C. A. (2002). School outcomes: sharing the responsibility between pupil and school. Education Economics, 10(2), 183-207

Thanassoulis, E., Kortelainen, M., Johnes, G. \& Johnes, J. (2011). Costs and efficiency of higher education institutions in England: A DEA analysis. Journal of the Operational Research Society, 62(7), 1282-1297.

Thieme, C., Prior, D., \& Tortosa-Ausina, E. (2013). A multilevel decomposition of school performance using robust nonparametric frontier techniques. Economics of Education Review, 32, 104-121

Thieme, C., Prior, D., Giménez, V. \& Tortosa-Ausina, E. (2011). Desempeño de los centros educativos: ¿Un problema de recursos o capacidades organizativas? Hacienda Pública Española, 199(4), 81-118. organizativas? Hacienda Pública Española, 199(4), 81-118.

Tran, C. D. T. \& Villano, R. A. (2018). Measuring efficiency of Vietnamese public colleges: An application of the DEA-based dynamic network approach. International Transactions in Operational Research, 25(2), 683-703.

Visbal-Cadavid, D., Martínez-Gómez, M. \& Guijarro, F. (2017). Assessing the efficiency of public universities through DEA. A case study. Sustainability, 9(8), 2-19. 
Worthington, A. C. (2001). An empirical survey of frontier efficiency measurement techniques in education. Education Economics, 9(3), 245-268.

Wang, D. D. (2019). Performance-based resource allocation for higher education institutions in China. Socio-Economic Planning Sciences, 65(1), 66-75.

Wößmann, L. (2003). Schooling resources, educational institutions and student performance: the international evidence. Oxford Bulletin of Economics and Statistics, 65(2), 117-170.

\section{AUTORES}

Alexander Arévelo Soto: magíster en Ciencias Económicas y de Gestión de la Pontifica Universidad Javeriana Cali. Actualmente docente e investigador tiempo completo del Departamento de Ciencias Básicas de la Institución Universitaria Antonio José Camacho, miembro del GISCBA y representante de la UNIAJC ante el IGCali. Correo: aarevalo@admon.uniajc.edu.co

Victor Giménez García: doctor en Ciencias Económicas la Universidad Autónoma de Barcelona. Actualmente docente e investigador tiempo completo del departamento de Empresa de la Universidad Autónoma de Barcelona y coordinador del área del operaciones y métodos cuantitativos. Correo: victor.gimenez@uab.cat

Diego Prior Jiménez: doctor en Ciencias Económicas la Universidad Autónoma de Barcelona. Actualmente docente e investigador tiempo completo del departamento de Empresa de la Universidad Autónoma de Barcelona y coordinador del área de finanzas, información financiera y control de gestión. Correo: diego.prior@uab.cat 